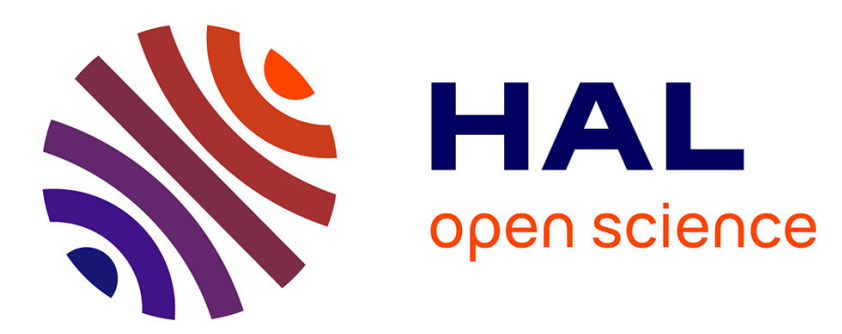

\title{
Augmenting Tactile 3D Data Navigation With Pressure Sensing
}

Xiyao Wang, Lonni Besançon, Mehdi Ammi, Tobias Isenberg

\section{To cite this version:}

Xiyao Wang, Lonni Besançon, Mehdi Ammi, Tobias Isenberg. Augmenting Tactile 3D Data Navigation With Pressure Sensing. Computer Graphics Forum, 2019, 38 (3), pp.635-647. 10.1111/cgf.13716 . hal-02091999

\section{HAL Id: hal-02091999 \\ https://hal.science/hal-02091999}

Submitted on 9 Apr 2019

HAL is a multi-disciplinary open access archive for the deposit and dissemination of scientific research documents, whether they are published or not. The documents may come from teaching and research institutions in France or abroad, or from public or private research centers.
L'archive ouverte pluridisciplinaire HAL, est destinée au dépôt et à la diffusion de documents scientifiques de niveau recherche, publiés ou non, émanant des établissements d'enseignement et de recherche français ou étrangers, des laboratoires publics ou privés. 


\title{
Augmenting Tactile 3D Data Navigation With Pressure Sensing
}

\author{
Xiyao Wang, ${ }^{1,2}$ Lonni Besançon, ${ }^{3}$ Mehdi Ammi, ${ }^{4}$ and Tobias Isenberg ${ }^{1}$ \\ ${ }^{1}$ Inria, France $\quad{ }^{2}$ Université Paris-Saclay, France \\ ${ }^{3}$ Linköping Universitet, Norrköping, Sweden ${ }^{4}$ Université Paris 8, France
}

\begin{abstract}
We present a pressure-augmented tactile 3D data navigation technique, specifically designed for small devices, motivated by the need to support the interactive visualization beyond traditional workstations. While touch input has been studied extensively on large screens, current techniques do not scale to small and portable devices. We use phone-based pressure sensing with a binary mapping to separate interaction degrees of freedom (DOF) and thus allow users to easily select different manipulation schemes (e.g., users first perform only rotation and then with a simple pressure input to switch to translation). We compare our technique to traditional 3D-RST (rotation, scaling, translation) using a docking task in a controlled experiment. The results show that our technique increases the accuracy of interaction, with limited impact on speed. We discuss the implications for $3 D$ interaction design and verify that our results extend to older devices with pseudo pressure and are valid in realistic phone usage scenarios.

CCS Concepts

-Human-centered computing $\rightarrow$ Interaction techniques; Visualization;
\end{abstract}

\section{Introduction}

Visualizions of 3D content are needed for exploring many types of scientific data, and good interaction design is a fundamental and essential prerequisite for effective visualization tools (e.g., [YKS07, Kee10]). As such, interaction research targeted specifically at data exploration plays an ever increasing role in the field of visualization (e. g., [Sut66, Hib99, Rhe02, Joh04, TM04, Kee10, KI13, Mun14, Ise16]). This need for effective and efficient interactive tools is evident, for instance, in the use of navigation for exploratory [Tuk77] 3D spatial data exploration [LKM*17]: by interactively changing the view, scientists are able to immerse themselves in the data [BCD*18] to understand its characteristics. Such tasks have long been performed on desktops using mice and keyboardsbut here the intuitive and fluent control of the exploration in 3D space is often challenging [FWSB07, BIAI17b]. Novel environments promise to better support 3D data exploration: they can improve the visual perception and spatial understanding using, e. g., wall-size screens [BI12], occluded VR glasses [QTHM06], and CAVEs [CNSD93] —often combined with dedicated input devices. Researchers also explore novel input metaphors for easy and precise control (e. g., touch-based [BWB06] and 3D spatial input [QM05]).

According to studies of different input metaphors for easy and precise control (e.g., touch-based [BWB06] and 3D spatial input [QM05]), tactile input ${ }^{\dagger}$ yields important advantages for 3D data exploration (e. g., [BIAI17b, CML*12, FGN10, Ise16, LRF*11, YSI*10]) such as faster completion times or increased 'directness' [BIRW19]. Moreover, the increasing use of smart phones makes this interaction easily accessible due to their good mobility and portability. Yet, mobile devices are restricted to a relatively small screen, possibly making the visualization of and the interaction with complex data impractical. Meanwhile, the emergence of visualization tools for mobile devices (e. g., Arctic Viewer, ImageVis3D, and KiwiViewer) ${ }^{\ddagger}$ suggests an increasing demand for mobile visualizations. We thus need to address the challenges of providing interactive data exploration control on these platforms.

Tactile interaction is the primary form of input on smart phones. Performed on a 2D surface, each touch point offers up to two degrees of freedom (DOF) through its translation, but navigation in 3D visualization requires six or more DOF to specify position and orientation [Ise16]. ${ }^{\S}$ To provide the necessary input DOF, existing techniques thus rely on 'multi-touch' input (e. g., [HCC07, RDH09]) or extra widgets (e.g., [YSI*10, ZF99]). More touching fingers or widgets on the screen, however, increase occlusion-a critical bottleneck, in particular, on the small surface of mobile devices. Another frequently used solution is 3D rotation-scaling-translation (RST) mapping: users control several integrated DOF [MCG10] with two fingers [LAFT12]. Although this mapping is popular, a recent study [BIAI17b] highlighted its difficulties: participants complained about the lack of separability of different DOF.

\footnotetext{
$\dagger$ We refer to touch input as 'tactile' input as elsewhere (e. g., [BIAI17b, BIAI17a, HW78, PM03, RGB* 10]), we do not mean haptic (tactile) feedback.

\$ https://kitware.github.io/arctic-viewer/, http://www.sci.utah.edu/software/ imagevis3d.html, and http://www.kiwiviewer.org/, respectively.

$\S$ Uniform scaling: 7 DOF, non-uniform scaling: 9 DOF (e. g., [CDH11]).
} 
We thus study the augmentation or combination of tactile input with other interaction paradigms to address its limits. In the past, tactile input has been combined with spatially aware tangible devices [SBD12, SvZP*16, BIAI17a], mid-air gestures [KKC*15], and pressure input (e. g., [BH09, HL12, CDVB17]) to offer more interaction possibilities. While these techniques have been positively evaluated, most of them rely on custom-made sensing units which limit their adoption. In fact, mobiles' built-in functions are far from fully explored. As one of them, touch pressure sensing ${ }^{\text {Tl }}$ is included in a number of recent phones, in this paper we leverage it for the control of different DOF for 3D data navigation. Specifically, we designed a pressure-augmented scheme to separate different DOF required for 3D navigation: we use force only to distinguish modes, not as a primary input. Our binary force mapping is easy to execute and to remember and takes inspiration from established tactile interaction mappings. We thus limit the number of touch-points to a maximum of two to leave as much as possible space for data display and allows users to precisely and independently control many of the DOF involved in 3D navigation. It also does not require the use of additional sensors on the mobile device/display (e. g., [HL11a, PN16, BAI17]).

Our contributions are thus threefold. First, we present the design of our pressure-augmented tactile navigation mapping. Second, we compare our approach to the established RST technique in a controlled study. We found that our technique increases the 3D manipulation accuracy in docking tasks, with only a small increase in interaction time. Finally, we discuss the usage of our technique on devices without pressure sensors based on pseudo-pressure and verify that our technique is also valid in realistic application scenarios.

\section{Related Work}

It has been recognized that efficient interaction design plays a key role in data visualzation tools, the trend of intergrating interaction and visualization research has been argued since 1966 [Sut66] until recent days [Kee10, KI13, Bes18, BCD*18, $\left.\mathrm{WBG}^{*} 19\right]$. We focus on the effects of combined touch and pressure input for improving 3D dataset navigation on mobile devices.

\subsection{Visualization on Mobile Devices}

While the visualization of complex datasets is generally well studied, most work has focused on workstations or environments with large displays. The popularization of personal smart phones and smart watches, however, allows us to put visualizations on small yet readily available displays, and researchers have begun to investigate small-display visualization settings (e. g., [LHD17, POR*18]). Mobile devices are also used as a props to interact with large visualization environments, from large screen [JDF12, BIAI17a] to $\mathrm{AR}$ environments [ $\left.\mathrm{SFH}^{*} 02, \mathrm{BBK}^{*} 06\right]$. In our work we augment a mobile's touch input to better support data navigation tasks.

\subsection{D Interaction with 2D Tactile Input}

The navigation in 3D datasets to gain information relies on frequent manipulations of view position and orientation. Due to the many

II Apple Inc.: '3D-touch'; Huawei Technologies Co. Ltd.: 'force-touch.' benefits of direct-touch input (e.g., [FGN10, LRF*11, CML*12, Ise16, YSI*10, BIAI17b]), it is now a widely supported means to control 3D environments. While the mapping of finger locations to manipulations is straightforward in 2D (e. g., [KCST05, $\left.\mathrm{HCV}^{*} 06\right]$ ), suitable mappings are less evident in 3D.

Early touch-based techniques like RNT (Rotate'N Translate) [KCST05] and RST [RDH09] are often used to control virtual objects in 2D space. Hancock et al. [HCC07] first extended RST to 3D and provided different mapping possibilities using up to three fingers to support 3D interaction, and later to offer a full 6 DOF Sticky Tools interaction technique [HtCC09] which maps two-finger motions to translations (along $x, y$, and $z$ ) and to a rotation around $z$. A third finger is used for rotations around $x$ and $y$. Reisman et al. [RDH09] also proposed 3D RST manipulations by using three or more fingers. Another idea frequently found for interactive visualization is to use screen widgets to select a certain manipulation: it reduces the number of fingers needed and preserves a high DOF count. Cohé et al. [CDH11], for instance, designed $t B o x$ which offered direct and independent control of up to 9 DOF by means of a virtual box around the 3D object. Yu et al. [YSI*10] proposed to reserve the display's borders for mode selection to control different manipulations. Increasing the number of fingers and using screen widgets is efficient in many cases. Yet, on small devices such as phones the former causes occlusion problems and a mismatch with common habits of interacting using at most two fingers, while the latter reduces the limited screen space that is needed for the visualization.

Reducing the number of fingers while keeping a high DOF count usually requires the integration of several DOF. For instance, Liu et al. [LAFT12] controlled 6 DOF with at most 2 fingers by integrating $3 \mathrm{DOF}$ ( $x$ - $/ y$-translation, $z$-translation, and $z$-rotation). Whether it is better to integrate or to separate the control of different DOF has been discussed in detail (e. g., [ZS97a,ZS97b, VCB09, MCG10]). Researchers found that it depends on the input device: only when many input DOF are available does it make sense to also provide integrated control [LKM*17]. With limited DOF of touch input it could thus be beneficial to provide separate DOF control. The Depth-Separated Screen Space [MCG10], for instance, showed that separating rotations and translations led to faster manipulations, while participants in another study [BIAI17b] frequently complained about DOF integration on touch screens. We thus further discuss the effects of DOF separation with the use of pressure.

\subsection{Augmenting Tactile Input}

Researchers also tried to combine tactile input with other input paradigms to get best of both worlds. Past research has investigated how to use internal sensor data to combine touch interaction with spatial/tangible interaction (e. g., [OO05, SvZP*16, SRE17, BIAI17a]). However, we generally discuss the use of another interaction paradigm to augment tactile input instead of being an additional primary input. Chen et al. [CSH*14], Withana et al. [WPSN15], and Hinckley et al. [HHP* 16] tried to combine pre-touch finger motions with touch input using different techniques. While these ideas have been positively evaluated, they rely on extra hardware.

In our work, we are interested in combing pressure sensing with tactile input. The lack of physical pressure sensing in commercial 
devices forced researchers in the past to use separate pressure sensors. Pelurson and Nigay [PN16] used the non-dominant hand to control pressure and augment navigation in large 1D data. Heo and Lee [HL11a] captured pressure from both sides and the back of a mobile and reported the difficult of maintaining pressure. They later introduced ForceDrag and force lock [HL12] to use pressure as an input modifier: pressure is only used to select the interaction mode before further manipulations are interpreted. They also suggested to use an indicator as virtual feedback. Heo and Lee's work inspires our own, but it is still limited to a small number of DOF which are not directly usable for 3D navigation. Besançon et al. [BAI17] captured finger pressure on the back of a tablet to control the interaction's gain factor. They thus used pressure to control an additional variable, and not to improve tactile interaction mapping. Recent work also investigated the use of direct pressure sensing for 3D manipulations. Wang et al. [WWX*18] designed a 3D positioning technique controlling 3 DOF with only one finger, providing depth information with pressure input. We are interested, in contrast, in using pressure sensing only to augment tactile input, such as to facilitate the control of $6 \mathrm{DOF}$ for different 3D navigation modes.

\subsection{The Use of Pseudo-Pressure}

Many mobile phones use capacitive sensing, yet without pressure data. Researchers thus explored pseudo-pressure that estimates the applied force based on contact area, temporal changes, or inertial sensors. Initial work assumed the contact area between finger and screen to increase with pressure, or postulated that a harder press usually lasts longer than a light tap. Benko et al. [BWB06] computed different contact sizes to distinguish the cursor's tracking and dragging state. Boring et al. [BLC*12] used contact area for mode selection. Arif and Stuerzlinger [AS13] exposed major challenges for pseudo-pressure: the contact area varies significantly between people and depends on the touch angle, while temporal approaches lengthen completion time. So they combined both touch time and its average surface. They later introduced an authentication system based on key sequences and pseudo-pressure [AMS14]. Heo and Lee [HL11b] used mobile phones' built-in accelerator and their detection algorithm distinguishes a gentle-tap and a force-tap. Goel et al. [GWP12] used vibration motor and gyroscope to measure the vibration absorbed by fingers. By using inertial sensors, these last two methods improved pressure prediction rate. In our work we first concentrate on physical pressure sensing but also investigate options to use pseudo-pressure sensing to use our techniques on more devices.And we take inspirations from previous pseudo-pressure based interaction mapping to bring forward our interaction design.

\section{Interaction Technique}

We start by discussing the design goals and resulting interaction designs for our pressure-assisted mode selection based on the mobile's pressure-sensitive screen, without extra sensors. Our general guidelines for supporting data exploration on small screens are as follows (partially from the literature [LODI16, NFRCC05]):

G1 To support effective data navigation, the interaction technique should support 6 DOF: 3 for $x$-, $y$-, and $z$-translation; and 3 for $x-, y$-, and $z$-rotation in perspective projection (or $x$-/y-rotation and uniform scaling in orthographic projection).
G2 To lower the learning cost and to keep the maximum of intuitiveness, the mapping should not differ too much from currently used techniques on touch screens.

G3 The screen space should be reserved for the visualization. The mapping should thus use as few widget/fingers as possible.

G4 New interaction techniques should provide as much interaction flexibility, preferably more, than existing ones.

Even though many existing techniques support a high-DOF manipulation, some rely on widgets that obstruct the view onto the manipulated visualizations. Alternatively, an RST-based input mapping can be used. The version of RST used most often for 3D manipulations on mobile devices [BIAI17b] works as follows: users perform rotations around the $x$ - $/ y$-axis by moving a single finger, translate along $x$ - $/ y$-axis by moving two fingers in parallel, rotate around the $z$-axis by rotating two fingers around a given point, and translate along the $z$-axis by pinching two fingers. With such a mapping, users can control all 6 DOF with a maximum of two fingers on the screen by integrating several DOF, without any widgets. We thus chose to combine pressure sensing with RST to fulfill our design goals G1, G2, and G3. Yet, RST is limitated because it always integrates $x$-/y-rotation with uniform scaling or $z$-translation, which can lead to a misinterpretation of user intents [BIAI17b]. We thus use pressure input to separate out the control of different DOF (supporting G4). Due to the known problems of using pressure input together with touch sensing (e.g., imprecise control [RBB04]), however, we want to treat it as different from other input types:

G5 Pressure should augment the touch input, not be a primary input. The pressure control should be as easy as possible to avoid additional workload or input errors.

G5 is important because it has been suggested that keeping a stable level of force during lengthy manipulations is too difficult [HL11a, HL12]. We thus use pressure sensing only to define spring-loaded modes $\|$ [Bux86, SKB92] in a quasi-postural [IH12] fashion. Similar to other work (e.g., [BLC*12, HL12]), this means that we evaluate pressure only at the start of any input motion to select an interaction mode. Once the mode is selected, the actual manipulations can be carried out with a normal force in all cases. This approach is more flexible than using time-based pseudo-pressure because users can reconsider/change their interaction intention after a finger is put down. Even though users can distinguish several discrete force levels [WSB10], a binary mapping (distinguishing only light and hard touch) is enough to provide the precise control that we need (having $6 \mathrm{DOF}$ and separating rotations from translation). It is also easy to perform since users do not need to worry about disturbing their interaction with accidentally high pressure input.

Based on these considerations, we use the following mappings (Figure 1) by default. A single-finger light touch initiates $x$-/y-rotations, while a single-finger hard touch starts $x$-/y-translations. A two-finger light touch starts a $z$-translation, similar to the widely accepted "pinching gesture"- - yet by not affecting the scale but the

|| Using spring-loaded modes means that a certain mode will be maintained, while users still keep the control (fingers still on the screen) [HGB*06].

** A dedicated menu can be called with a three-finger touch on the screen, a gesture which we do not used for 3D manipulation. 


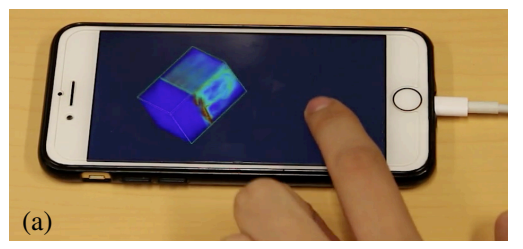

(b)

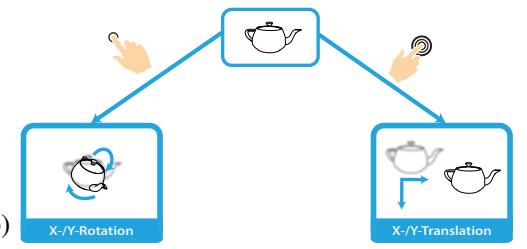

(c)

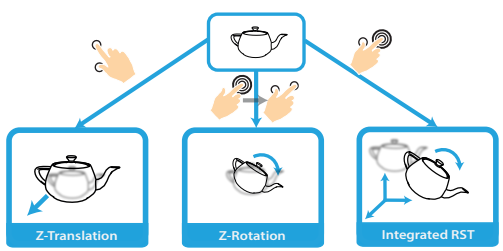

Figure 1: (a): touch+pressure manipulation of 3D data. Interaction mapping: (b) one-finger motions and (c) two-finger motions.

\section{()}

(a)

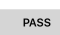

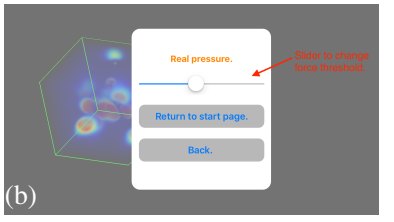

Figure 2: Force threshold setting: (a) initial calibration, users touch at specific positions; (b) slider-based adjustment.

distance to the camera. For rotations around the $z$-axis, finally, a user first performs a single-finger hard touch and then puts down the second finger to start the manipulation. To avoid unwanted translations (the first finger may move a bit before the second finger is put down), we initialize a timer: if the second finger is put down shortly after the hard touch is performed (we use a threshold of 1 second, based on our pilot studies), we consider that the translation of the first finger is undesired due to mis-operating, then the data will be reset to the status before the first touch is effected. In contrast, if the second finger is put down after the time-out we treat the finger's translation is deliberate and keep the data. The exact mapping could be different according to the specific dataset: for exploring some datasets, translations are more important than rotations, while rotations are key for others. We would map more important motions with light pressure (lower effort), but keeping the overall interaction design. As study the general use of pressure, we do not assume any specific type of data, so our specific mapping should be considered as an example. Finally, we add an integrated two-fingers gesture: putting down two fingers at the same time, with either one being a hard touch, is mapped by default to integrated RST. Aside of this last mapping, our design thus allows users to separate the translations from rotations. Interestingly, we thus go further than Martinet et al. [MCG10] who studied the effect of separating translations from rotations-we also separate between the $x$ - $/ y$-axes on the one hand and the $z$-axis on the other hand to understand the impact of a further increase of the separability of DOFs. However, our study is different from theirs as we separate the DOFs with an other input paradigm, and use a different mappings.

We derived this overall mapping based on past experience with touch-based 3D interaction, and the two-level pressure input is likely to be generally applicable. Its specific parameterization, however, will likely depend on people's personal preferences as well as on differences between devices. We thus formulate our next goal as

G6 The choice of different pressure levels should be adjusted for different users and different devices.

To realize this calibration, we distinguish between a light and a hard touch with a threshold $\alpha$. We perform an initial calibration to ensure
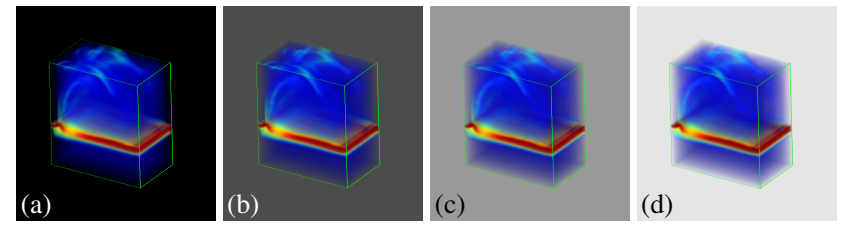

Figure 3: Example of visual feedback for volumetric flow data: (a) no touch; (b) light mode; (c) hard mode; and (d) integrated RST mode. We map brighter colors to more force and only change the brightness, not hue, to avoid misperception.

that this threshold is suitable for each user (Figure 2(a)). To account for different finger angles [BLC*12,RLG09], we record the pressure at several different positions. We average the maximum pressure value that was applied for a single touch gesture in each mode and thus derive $\alpha$ as the midpoint between both pressure averages:

$$
\alpha=\frac{\frac{1}{n} \sum_{i=1}^{n} \operatorname{HardMax}+\frac{1}{n} \sum_{i=1}^{n} \text { LightMax }}{2} .
$$

We later validate this model of using an averaged single $\alpha$ over the full screen with a small study (Section 7). A manual adjustment of $\alpha$ is also possible later (Figure 2(b)), ${ }^{* *}$ the initial position of the slider is associated with a calibrated threshold. In addition to calibration, it is also essential to make users aware of their input. We thus state

G7 The pressure level has to be evident for effective control.

The importance of visual feedback for pressure input has been argued by previous work (e. g., [RBB04, HL11b, HL12]). For example, Ramos et al. [RBB04] recommend "real-time [and] continuous feedback." Since our users do not need to maintain the pressure after a mode has been selected, visual feedback is all the more important. It is the only way to show users their selected interaction mode. We use background color to indicate the current mode, instead of a scroll bar or text to avoid occlusion. It maintains maximum data visibility (to support both G3 and G7). The color can be chosen according to each dataset's properties to ensure that it is not in conflict with the visualization. For example, Figure 3 illustrates our choice for visualizing volumetric flow data. Yet, to navigate in large or compact datasets, a common approach is to zoom into the data [CML*12]. In such cases all view space is filled by the data itself, our visual feedback does not have any effects. We thus also added haptic feedback with a short vibration when passing from light to hard touch mode.

We implemented our interaction technique on iOS: we load 3D data with $\mathrm{C}++$ and render it with OpenGL ES 3.0 using our own shaders. We use the VTK 7.0 framework to support some specific scientific datasets (*.vtk, *.vti). We capture and process input events 
and related data (e.g., touch position, pressure, gesture) with Swift 3 and iOS SDK, and translate the input to change the data view.

\section{Experiment}

To evaluate our technique, we wanted to compare it against other tactile $3 \mathrm{D}$ techniques. We excluded techniques using more than two fingers (e. g., [HCC07, RDH09]) because of the occlusion issue that we aimed to avoid. We also did not consider techniques that use screen widgets or interaction zones (e. g., [CDH11, YSI*10]) because they further reduce the available display size. We thus decided to compare our approach with the frequently used [BIAI17b] 3D-RST technique described in Section 3.

The goal of our experiment is thus to understand the effects of using pressure for separating DOF for 3D navigation tasks, based on quantitative data as well as qualitative feedback and observations from the use of both techniques. One of our reasons for separating interaction DOF was to increase interaction accuracy, so we also wanted to test whether the use of different gain factors (i. e., controldisplay ratio, CD) had an impact on the performance: With a high gain factor, a small user input results in a large movement, and unwanted operations due to RST's integrated DOF thus potentially result in a higher frustration. In contrast, a low gain factor may result in these unwanted operations not being noticed.

\subsection{Design}

We wanted to compare how participants perform 3D rotations and translations with both techniques. However, generic navigation in large 3D datasets is difficult to control. We thus chose a 3D docking task which comprises translations in $3 \mathrm{DOF}$ and orientations in 3 DOF. A docking task consists of bringing a virtual object to a target position and orientation. Such docking tasks are common in the 3D interaction literature [BAI17, BIAI17b, CMS88, GBC13, HCC07, HPGK94, IBG*16, VSC15,Zha98]. We used the Stanford bunny** - as done in previous work [BAI17, IBG*16]—due to its easily understood shape without orientation ambiguity. Our docking target was transparent green, while the object to dock was opaque white. While our technique allows participants to also make use of the RST's integrated DOF (Figure 1(c), bottom-right), we wanted to better understand the advantages and limitations of both approaches (i. e., integrated and separated). We thus removed, only for the experiment, the possibility to use the integrated DOF with our technique.

Our experiment has two independent variables ( 3 gain factors and 2 techniques) and we measure two dependent variables (completion time and accuracy; see Section 5.2 for our method of measuring). In addition to the factor of 1 (a 1:1 mapping), we checked for effects of higher (2) and lower (0.5) gain factors. Our experiment thus used a within-subjects design with a total of 6 conditions. To account for variability we used 12 trials per technique per gain factor, resulting in 72 trials per participant. We counter-balanced the trial order using a Latin square to balance learning biases and tiredness. We also validated the target's positions and orientations from randomly generated targets by removing those that were hard to reach, resulting in the same pool of target positions for all participants. We varied the initial angle difference between the object and target from $27^{\circ}$ to $180^{\circ}$ (mean of $122.2^{\circ}$ ) and the initial distance difference between 5150 and 7076 units (in virtual space scale; mean of 6433.9).

\subsection{Participants and Apparatus}

We recruited 24 unpaid participants ( 8 female; ages $22-53$, mean 30.9 , med 26, SD = 10.4). 19 had at least a bachelor degree, while 5 had at most an A-level equivalent. 16 were experienced with 3D manipulation through extensive use of video games or 3D modeling software and 7 of them reported frequent use (daily/weekly). All of them reported to use tactile interaction daily on smart-phones or tablets, while 6 of them have used other tactile input devices. All had normal or corrected-to-normal vision. 1 participant was left-handed.

We ran the study on an iPhone 7 (4.7" screen diagonal, $750 \times 1334$ pixels, 326 ppi, iOS 10.0.0). During the test, the smart phone rested on the table, in landscape mode. While this apparatus does not exactly copy the use of smartphone which are generally handheld, such controlled experiment setups are not uncommon in the field of HCI and visualization with, for instance, non-wrist-worn smartwatches [BBB*18, BBB*19] or touch-based systems [DJFW15, KH11]. We decided to also conduct our study in a similar controlled/constrained way to remove possible confounds (e. g., both how the phone was held by participants and which finger they used could introduce additional noise to the pressure control). Moreover, we discuss later in Section 7 how our results still generalize to normal phone use.

\subsection{Procedure}

We told our participants that we would ask them to perform 3D manipulations on the phone that rested on the table in landscape orientation. To avoid participant response bias [DVM*12], we told them that both methods were state-of-the-art, that none of them was invented by us, and that we wanted to compare their performances.

We began with an initial calibration for $\alpha$ (as described in Section 3). We then told participants that they would use one technique first, explained how it worked, and asked them to perform as many training trials as they wanted with different gain factors. We asked them to take good advantage of the training to get familiar with the technique. When participants reported that they were ready, we started the trials. Participants began and validated each trial by touching a button on the corner of the screen. We asked them to balance speed and accuracy but did not reveal their achieved accuracy immediately to avoid a bias toward accuracy [HPGK94]. When they finished all trials for one technique, we introduced the other technique and repeated the process (unlimited training and tests).

We also asked them to fill in questionnaires. Participants reported their age, education background, and how familiar they are with tactile screen and 3D manipulations, before we introduced the task. After each technique, we asked them to fill in a form to assess their workload (NASA's Task Load Index TLX ${ }^{\dagger \dagger}$ ) and their fatigue (based on Shaw's approach [Sha98]). To avoid seemingly random choices made in the second part of the TLX (often seen as confusing by participants in our pilot studies) that would lead to inconclusive or incorrect results, we removed the second part of the TLX questionnaire to perform a $R A W T L X$. It is, according to Hart's survey [Har06], equally well suited as a regular TLX. At the end of the experiment, we asked all participants to give us their overall preference (the techniques could be named to be equal) as well as ad- 
vantages and drawbacks that they experienced with both techniques. We recorded think-aloud comments throughout the study.

\subsection{Hypotheses}

Based on our pilot studies and results reported in previous work, we formulated a number of hypotheses:

H1 Both techniques exhibit a similar performance (accuracy and time) overall as the interaction mappings are close to each other. We do not believe that the separated DOF has a strong impact on accuracy in general but,

H2 we hypothesis that, with a higher gain factor, DOF integration result in worse performance and we thus expect RST to yield lower accuracy scores than our technique.

H3 The overall workload is identical for both technique. While the frustration is higher with the RST due to the integration, the mental workload is probably lower because this technique is already frequently used.

H4 The force-touch technique increases the overall fatigue, probably with increased finger and hand fatigue.

\section{Results}

We collected a total of 1728 docking trials from our 24 participants. In addition, we recorded self-stated feedback and answers to subjective preference questions. While data from HCI experiment has usually been analyzed by the NHST(Null hypothesis significance testing) in the past, we choose to report our results using estimation techniques with effect sizes and confidence intervals instead, as recommended by the APA [Van09] and also because the former approach is being increasingly criticized by statisticians [Lai10, Cum12, Cum13, Cum14, VAL15, Bak16], stats practitioners [AKNR17], and HCI researchers [DCH14, Dra16, BD17, BD19]. While dichotomous interpretations based on $p$-values are still extensively used [BD19], approaches relying on effect sizes and more nuanced interpretations are now widely recommended [Cum 12 , Cum13, Cum14, VAL15, Dra16, BD19]. However, it is still possible to read our results based on $p$-value by comparing CIs spacing with common $p$-value spacing [KA13]. We detail most of the results in this section by technique and by gain factor to better understand the latter's potential impact on the former.

\subsection{Completion Time}

We analyze log-transformed time measurements to correct for positive skewness and present, in Figure 4(a), anti-logged results with geometric means that dampen the effect of extreme completion times that would bias an arithmetic mean, as recommended in such cases [SL10]. \# Our results show that, overall, there is strong evidence that RST is faster than our technique. For normal (i.e., 1) and low (i. e., 0.5) gain factor values, there is weak evidence that

\footnotetext{
** https://www.cc.gatech.edu/ turk/bunny/bunny.html

†† http://humansystems.arc.nasa.gov/groups/tlx/downloads/TLXScale.pdf

$\dagger \dagger$ Arithmetic means use the sum of a set of values, while geometric means use the product of the set's values.
}

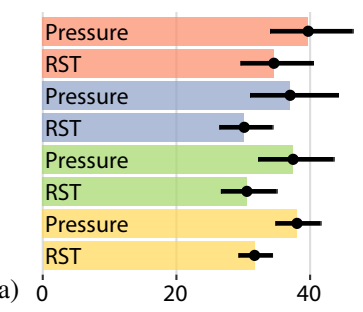

(b) 0.0

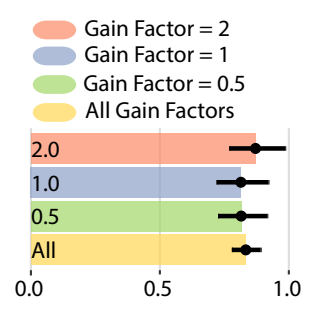

Figure 4: Task completion time: (a) absolute values and (b) pairwise ratios. Error bars: $95 \%$ bootstrapped CIs.
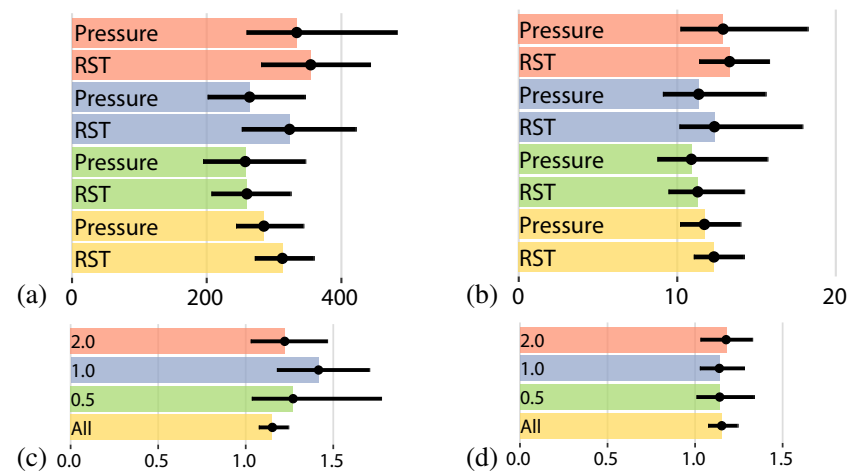

Figure 5: Accuracy: (a),(c) Euclidean and (b),(d) angular distances, (a),(b) absolute values and (c),(d) pair-wise ratios. Error bars: 95\% bootstrapped CIs. Colors as in Figure 4.

this faster completion time still holds, while for high (i. e., 2) gain factor values our data would suggest that there is no difference. We also report on the effect size in Figure 4(b) which corresponds to pairwise ratios, ${ }^{\S \S}$ available at https: //aviz . fr/ci/ [BD17].The non-overlap of each confidence interval with the value of 1 clearly shows strong evidence that the completion time is shorter with the RST technique than with our technique in all cases except the 2.0 gain factor value condition. For this one, the evidence is still strong, however. For RST, overall, participants needed a bit longer than $4 / 5$ of the time needed for our technique, so the overall difference in completetion time is not large.

\subsection{Accuracy}

We determined the Euclidean and angular distances between the manipulated object and the docking target when the participant validated a trial (Figure 5), using the objects' centers for the former

$$
d_{e}=\sqrt{\left(x_{t}-x_{o}\right)^{2}+\left(y_{t}-y_{o}\right)^{2}+\left(z_{t}-z_{o}\right)^{2}}
$$

and deriving the angular difference as

$$
d_{a}=2 \cdot \arccos \left(q_{d \omega}\right) ; \quad q_{d}=q_{o}^{-1} \cdot q_{t}
$$

with $q_{o}$ as the manipulated object's quaternion, $q_{t}$ as the target's quaternion, thus $q_{d}$ being the difference quaternion, and $q_{\omega}$ be-

$\S \S$ Pairwise ratios are computed for each individual subject, i. e., to devide measurement per each individual subject. Our computation scripts are 

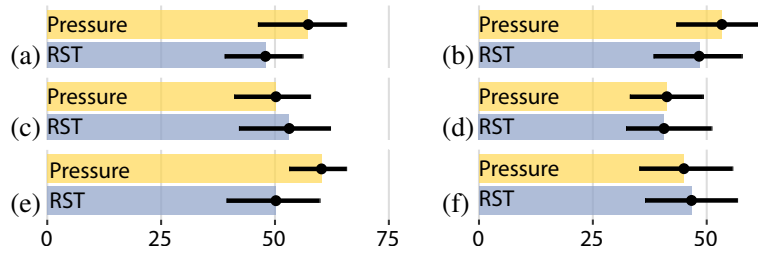

75

Figure 6: Workload in TLX units (lower is better) for (a) physical, (b) mental, and (c) temporal demand, (d) performance, (e) effort, $(f)$ frustration. Error bars: $95 \%$ bootstrapped CIs.
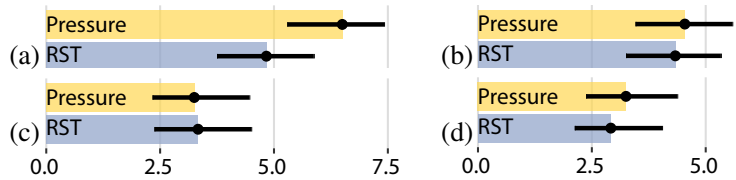

7.5

Figure 7: Fatigue measurement for (a) fingers, (b) hands, (c) arms, and $(d)$ shoulders. Error bars: $95 \%$ bootstrapped CIs.

ing the $\omega$ component of an $\omega+x i+y j+z k$ quaternion with $i^{2}=j^{2}=k^{2}=i j k=-1$. The overlapping Confidence Intervals (CIs) in Figure 5(a) suggest that with our data we cannot find evidence of a difference of Euclidean distance between the two techniques. We computed the ratio RST/Pressure for each gain factor to look at the intra-participant difference. The non-overlap of each confidence interval with the baseline 1 provides strong evidence for our technique being more precise than the classical RST mapping w.r.t. Euclidean distance. This observation is strongest for a gain factor of 1: our technique is almost 50\% more precise than RST. Similarly, the CIs in Figure 5(b) do not provide evidence of a difference between the two technique for angular distances. However, none of the confidence intervals overlaps with 1 in Figure 5(d) so our technique is overall more precise than RST.

\subsection{Measuring Workload and Fatigue}

We report the TLX results in Figure 6. The mental and temporal demand (Figure 6(b), (c)) as well as frustration and performance ratings (Figure 6(f), (d)) show no evidence of a difference between both techniques. Even though the confidence intervals are overlapping, a difference may exist for physical demand: our technique's physical demand (Figure 6(a)) could be higher than that of RST. The even smaller overlaps of the confidence intervals for effort (Figure 6(e)) suggest that participants thought it was higher for our technique than for RST. Overall, however, the two differences are small and it appears that the general workload is similar for both techniques.

We report fatigue data in Figure 7. The large overlap of CIs for shoulder (Figure 7(d)), arm (Figure 7(c)), and hand (Figure 7(b)) shows that we did not see a difference between the two techniques. A higher finger fatigue caused by our technique, however, is highlighted by the smaller CI overlap in Figure 7(a).

\section{Discussion}

\subsection{Completion Time}

We have gathered with our experiment evidence against $\mathbf{H 1}$ because we did notice a difference in completion time between both techniques. It appears through our results that RST can be a bit faster than our technique, thus partially contradicting previous studies [MCG10, NBBW09]. This difference of results can probably be explained by two factors. First, many users are already aware of the gestures used in RST mapping for 2D manipulations on mobile devices. When it comes to our technique, we observed that users chose to take only 2-3 minutes and, at most, 5 minutes for training. This reduced learning (compared to the long experience with 2D RST) resulted in the need to try to recall the interaction mapping during the experiment. This recall leads to slower interaction, which is common with any new technique as explained by MacKenzie and Zhang [MZ99] (elusive crossover point). Second, the tight DOF integration of RST may also contribute to the faster completion times. Indeed, integrated DOFs allow participants to perform several manipulations in short and fluid succession, which would have to be executed sequentially with our technique. While we believe that this sequential interaction is an asset for accuracy purposes, and the slowing down is likely not a problem for our complete interaction mapping which still provided integrated DOF manipulations as explained in Section 3.

\subsection{Accuracy}

Our accuracy results provide evidence to partially validate $\mathbf{H 2}$. While we wrongly believed that the accuracy would be much more different for a higher gain factor, it remains that, overall and for all gain factors in particular, the accuracy was better with our technique than with the RST mapping. This result can also be explained by the sequential manipulations that are impossible to achieve with the RST mapping, thus making small modifications of single DOF almost impossible. This is where our technique shines. While it was not critical for a docking task, such an accuracy is very likely to be fundamental in many scientific domains which rely on 3D data visualization and precise interaction.

\subsection{Workload and Fatigue}

Our hypothesis $\mathbf{H 3}$ seem to be validated by our experimental data, though our reasoning was wrong. The overall workload seems to be similar for both technique, thus highlighting that having this additional input to augment tactile interaction was not hindering users' workload. The mental workload results clearly highlight the fact that the learning needed to master our technique was not an issue. However, this additional effort in learning probably influenced the effort evaluation done by our participants. This idea is further reinforced by the fact that seven participants actually reported it. One could thus wonder whether, with a longer exposure to pressurebased mode selection, this effort would not show a tendency that is more similar that of RST.

Based on our pilot studies we expected our approach to increase finger and hand fatigue, thus increasing the overall fatigue of our technique compared to the RST mapping. This assumption was partially right: the finger fatigue is indeed increased by the use of our technique, but the hand fatigue measurements are the same for both techniques. This increase in the finger fatigue was easy to predict, as users have to push harder on the screen more often. However, one can notice that the finger fatigue is not much higher 
with our technique, and that no participant later complained that their finger was hurting, even though the total duration of the study was of 44.6 minutes on average ( $24.6 \mathrm{~min}$ for our technique, a bit more than RST). One should take into account that the overall manipulation time was longer with our technique than with the RST mapping. While this is only a few seconds per trial, it easily represents several minutes when multiplied by the 36 trials per technique. We thus believe that the gained accuracy obtained with our technique is probably worth a bit of extra finger fatigue. Furthermore, the fact that some of our participants had not been exposed to such pressure interaction before did contribute to the higher fatigue: they usually pressed harder than needed to switch modes—-such behavior would likely quickly disappear with a longer exposure to our technique.

\subsection{Preferences and Qualitative Feedback}

Overall, 14 participants preferred RST, judging it faster and/or more natural, while 9 participants reported our technique as their favorite one. One reported that he liked both equally. None was evaluated as inappropriate for the task. This result contradicts those of Nacenta et al. [NBBW09] for 2D interaction and Martinet et al. [MCG10] for $3 \mathrm{D}$ interaction that integrating DOF could reduce user satisfaction. We believe that this disparity can be explained by the fact that RST is frequently used for 2D or 3D applications [BIAI17b]. Moreover, the studies by Martinet et al. [MCG10] and Nacenta et al. [NBBW09] were conducted in 2009 or 2010. Since then, RST established itself as predominant. Such a long-term exposure is likely to bias subjective ratings [BS71], and is thus likely to have also biased our participants- 16 of them reported to be familiar with 3D manipulations and have surely been exposed to RST before, at least in its 2D form. Moreover, five participants stated that our technique specifically needed to be learned since pressure interaction is but still rarely used today, while RST was deemed as natural and easy to understand by 11 participants. Still, nine participants stated that our technique, though difficult at first, could be understood easily, and once mastered would provide just as good results. The preference for the RST could also possibly be explained by the fact that participants were faster with it. Four participants actually stated so. Taking into account the high number of docking tasks they had to perform, it is likely that there could be a bias toward the fastest technique.

Our fatigue results are also reinforced by participant comments: ten reported that they could feel, at some point, the extra effort on their fingers. This fatigue was probably emphasized by the fact that, for some participants, first pressing hard and then releasing was not natural (two mentioned it). Some continued to press hard throughout all manipulations - spring-loaded modes are also rare in public applications and possibly have to be learned. Nonetheless, participants also highlighted our findings on accuracy. Sixteen of them praised our technique for its better accuracy over RST and reflected on the fact that it helps avoid unintentional DOF manipulations.

Interestingly, three participants commented on the occlusion issue and mentioned, while performing, that using two fingers for translation (i. e., RST) was problematic because a good part of the screen was hidden. They mentioned that this problem was solved with our technique. This occlusion reduction is an improvement over the RST mapping: most of the interaction (rotation around and translation along the $x / y$-axes) can be done with a single finger, while the RST technique requires 2 fingers for all but $x / y$-rotation.

Even though the learning issue was mentioned by nine participants for our technique in contrast with the 'natural' mapping of RST, participants mastered our technique. This finding is also supported by the similar mental workload scores for both techniques and that no participant reported that our technique was too hard to understand, three of them even reported that it was easy to use.

Three participants reported that our visual and haptic feedback helped them. We are still unsure whether haptic feedback would be enough to differentiate between two values only. Since most mobile applications today provide notifications through haptic feedback, however, it does not seem unreasonable to keep a visual feedback.

Though they could change the force feedback and we explained why this is useful, none of our participants felt the need to adjust it. This observation suggest that our initial calibration phase, while simple, is sufficient to calibrate front-of-device input for two values.

Finally, we also noticed an interesting use of the two-finger interaction. Seven of our study participants switched between one-handed interaction and two-handed interaction, even on such a small device. This possibility was probably exacerbated by our setup since users did not have to hold the device at all, thus having both hands free for interaction.

\subsection{Limitations and Future Work}

With our study we evaluated the potential of our pressure-augmented tactile interaction technique on a smart phone for 3D data navigation, in particular to separate out the different DOFs (translations from rotations). While we paid attention to as many factors as we could (e. g., several gain factors), there are other unevaluated aspects. For instance, our study was only done on a single screen size and we can conjecture that the screen size factor could be responsible for an increase/decrease in the occlusion issue as mentioned by our participants. Although there is no reason that our technique cannot be scaled to larger screens, the potential fatigue requires reflection on how to reduce it. A higher gain factor could, for instance, avoid long dragging on large screens.

Similarly, it would be interesting to check whether our technique could be easily remembered and used. We wonder whether our participants would have been faster and/or more accurate after a longer exposure to our technique. Indeed, comparing our mapping to RST was somewhat unfair since most people actually know and understand the RST mapping. As a consequence, we would like to investigate how much better our pressure-based technique could be used with a proper and longer learning phase.

With our design, pressure is used only at the beginning. It would thus also be interesting to investigate if pressure could be used in other phases (e. g., to change interaction mode during the manipulation to avoid frequent shifting of fingers, or to validate/cancel the manipulation at the end).

Docking tasks are abstract, yet they are generalizable to many tasks in 3D [BIAI17b, CMS88, HPGK94]. Even though some interaction intents in 3D visualization go beyond this paradigm, specific tasks (such as 3D subset selections, 3D points manipulations 
Table 1: Results of pseudo-pressure pilot test.

\begin{tabular}{lcccrc}
\hline feature & $\begin{array}{c}\text { true } \\
\text { positive }\end{array}$ & $\begin{array}{c}\text { false } \\
\text { positive }\end{array}$ & $\begin{array}{c}\text { true } \\
\text { negative }\end{array}$ & $\begin{array}{c}\text { false } \\
\text { negative }\end{array}$ & accuracy \\
\hline T(time) & 121 & 15 & 225 & 119 & $72.1 \%$ \\
R(radius) & 190 & 57 & 183 & 50 & $77.7 \%$ \\
T and R & 108 & 12 & 228 & 132 & $70.0 \%$ \\
T or R & 203 & 60 & 180 & 37 & $79.8 \%$ \\
\hline
\end{tabular}

and specifications, temporal navigation, data read-outs, etc. [KI13]) could still take inspiration from our initial work. For example, the slicing of volumetric data also requires the precise control of the slicing plane's position and orientation. We thus would like to further investigate how pressure could be used in such demanding contexts. While our study exclusively investigated mobile device use only, such devices can also play a role in larger visualization environments as we noted before. It would thus be interesting to study the use of our technique in combination with such environments.

Finally, the prototype we used in our study can naturally be improved. For example, there could be better ways to trigger the menu, we can improve the visual and haptic feedback, and we could adjust the way to manage the exact interaction mapping of the pressure input (e.g., which pressure level triggers what motion), etc.

\subsection{Extension to Older Devices with Pseudo-Pressure}

To generalize our approach to devices without physical pressure sensing, we can make use of pseudo-pressure sensing discussed in Section 2.4. High-accuracy prediction techniques (e. g., [HBW13, GWP12]) usually trigger vibration when a touch is detected. This can disturb normal manipulation and is not compatible with our haptic feedback. We thus investigated previously envisioned and simpler features such as contact area or touch completion time [AMS14, AS13, BWB06, BLC*12]. Indeed, we also observed that the contact area increases with the pressure as described by Arif and Stuerzlinger [AS13] and users begin to move their finger(s) very quickly when performing a light touch motion, while a hard mode manipulation exhibits a longer time before fingers start to move. We propose to establish our model based on these two features. We then started by conducting a pilot test to parametrize the hybrid model. We asked the same 24 participants to first calibrate the touch sensing by performing five one-finger translations in both light and hard mode. Initial touching target and ending translation targets are shown each time differently on the screen. We computed the average time $t$ that the finger takes to move more than a given distance $d$ (five pixels in our experiment) for both modes, and used the midpoint value for the time threshold. If the finger stays longer than the threshold without shifting outside $d$, we assume that the time criteria is attained to be classified as a hard touch. Similarly, we computed the radius threshold by using the middle value of the average contact radius. After calibration, we asked each participant to perform one-finger translation 10 times in each mode, with the order and touch position as well as the trajectory being randomly selected from a pre-defined data pool. We gathered 480 one-finger motions (Table 1). Our prediction accuracy is low when compared to the good accuracy of proper pressure sensors (up to $97 \%$ for binary level [GWP12]). Also, the SDK of iOS 10 does not allow us to directly obtain the continuous contact area on the screen but rather gives us one of the few possible discrete values. However, our captured data showed us that it may be possible to create more precise models. We observed that a hard touch has a peak in its contact area data several milliseconds after the initial touch is made and then suddenly drops. Relying on this, we could improve our accuracy to make our technique efficient on non-pressure sensing devices in the future.

\section{Realistic Usage of Phones}

To verify that our technique could also be used in a non-controlled setup when people are holding the phone, we conducted a second test to see if different touching areas and different holding postures would influence pressure. We divided the screen uniformly into nine areas ( 1 is top-left, 9 is bottom-right), and asked participants to touch these areas both normally and hardly, four times each, in a randomized order, guided by an indication on the screen. We used a within-subjects design with a total of 4 counter-balanced conditions ( 2 different hands (left and right) $\times 2$ postures (phone on the table and phone held by participants)). We told participants to perform the touches only with their index finger as seems to be usual in most 3D applications on mobile devices [BIAI17b]. We recruited 8 unpaid volunteers, all right-handed. We ran the experiment on the same device as used in Section 4. We gathered 2304 touch events $(8$ participants $\times 2$ hands $\times 2$ postures $\times 9$ areas $\times 2$ pressure levels $\times$ 4 repetitions), see Figure 8. The overlapping CIs in Figure 8(a) indicate that pressure for light touch, hard touch, and average threshold $(\alpha)$ is similar with both hands. While the overlapping of CIs in Figure 8 (b) provides evidence that pressure for light touch, hard touch, and average threshold $(\alpha)$ differs according to the mobile's postures, we can see that the absolute value difference is low. The calibration technique we used thus scales to the phone-holding posture. The small value difference can also be compensated by manually adjusting the threshold. The CIs in Figure 8(c) show strong evidence of differences between zones for similar pressure input (e. g., between $7,8,9$, and 1). However, Figure 8(c) also shows that it is still possible, for all areas on screens, to distinguish between a hard and a light touch. Based on this data, we are thus confident that our technique scales to realistic hand-held phone usage.

\section{Conclusion and Perspective}

We reflected the use of mobile pressure sensing and presented an interaction mapping to separate different DOF for 3D navigation. This new type of interaction design and input mapping allows us to provide effective visualization exploration tools on new platforms such as mobile phones that have become ubiquitous in today's world yet which have input capabilities and output constraints fundamentally different from established data exploration platforms such as workstations. With our technique, we can reduce, in particular, the occlusion issue often experienced with more-than-two-finger techniques on small devices, do not have to resort to the integration of several DOF, and can use the entire screen space for data visualization. In our experiment we determined that our technique is more precise than the integrated RST technique which is common in the literature and in mobile applications, with limited impact on speed. 


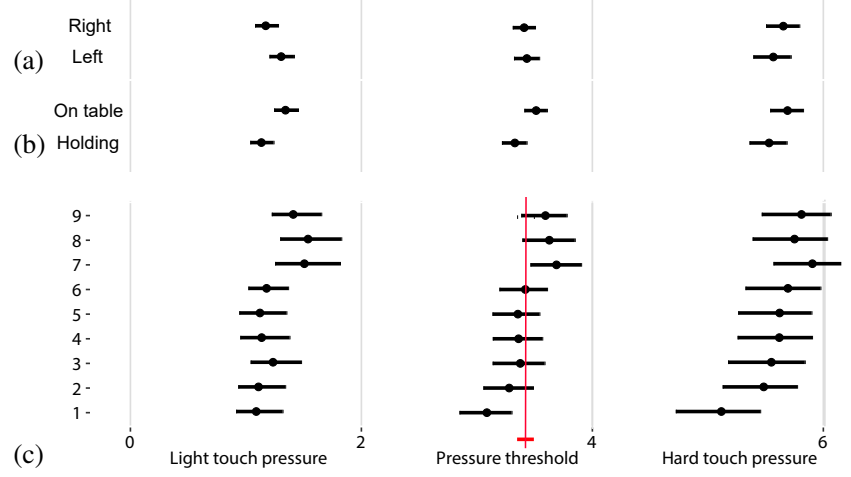

Figure 8: Pressure for different study conditions: (a) left and right hand; (b) holding the phone and put it on table; (c) different areas, the red line is the threshold over all screen. Error bars: $95 \%$ bootstrapped CIs.

An important insight from our study is that pressure-based springloaded moding in quasi-postural fashion is easily understood, used, and does not increase users' mental workload. Also important is the fact that our results and our participants' feedback confirmed that such a separation of DOF leads to a better accuracy. These results can be of critical importance for some specific scientific domains which rely on interactive visual data exploration to gather insights.

Finally, we reflected on the possibility to use our technique with devices without force input. While we conducted our experiment in a controlled environment to reduce possible confusion, we also verified that our pressure calibration model can be directly applied to realistic application scenarios.

\section{Acknowledgments}

We thank all our study participants for their time and feedback. We also thank all people from our Aviz project team who provided feedback about previous versions of this article.

\section{References}

[AKNR17] AMrheIN V., KORNER-NIEVERGELt F., Roth T.: The earth is flat $(p>0.05)$ : Significance thresholds and the crisis of unreplicable research. PeerJ 5 (July 2017), e3544:1-e3544:40. doi: 10.7717/peerj.3544

[AMS14] Arif A. S., MazaleK A., Stuerzlinger W.: The use of pseudo pressure in authenticating smartphone users. In Proc. MOBIQUITOUS (2014), ICST, Brussels, pp. 151-160. doi: 10.4108/icst.mobiquitous. 2014.257919

[AS13] ARIF A. S., StUerzlinger W.: Pseudo-pressure detection and its use in predictive text entry on touchscreens. In Proc. OzCHI (2013), ACM, New York, pp. 383-392. doi: 10.1145/2541016.2541024

[BAI17] BesAnÇON L., Ammi M., Isenberg T.: Pressure-based gain factor control for mobile 3D interaction using locally-coupled devices. In Proc. CHI (2017), ACM, New York, pp. 1831-1842. doi: 10.1145/3025453. 3025890

[Bak16] BAKER M.: Statisticians issue warning over misuse of $P$ values. Nature 531, 7593 (Mar. 2016), 151. doi: 10.1038/nature.2016.19503

[BBB*18] Blascheck T., Bezerianos A., BesAnÇON L., LeE B., ISENBERG P.: Preparing for perceptual studies: Position and orientation of wrist-worn smartwatches for reading tasks. In Proc. CHI Workshop on Data Visualization on Mobile Devices (2018).

[BBB*19] Blascheck T., BesAnÇON L., Bezerianos A., LEe B., ISENBERG P.: Glanceable visualization: Studies of data comparison performance on smartwatches. IEEE Transactions on Visualization and Computer Graphics 25, 1 (Jan. 2019), 630-640. doi: 10.1109/TVCG.2018. 2865142

[BBK*06] Bornik A., Beichel R., KruijfF E., Bernhard R., SCHMALSTIEG D.: A hybrid user interface for manipulation of volumetric medical data. In Proc. 3DUI (2006), IEEE Computer Society, Los Alamitos, pp. 29-36. doi: 10.1109NR.2006.8

[BCD*18] Büschel W., Chen J., Dachselt R., Drucker S., Dwyer T., Görg C., Isenberg T., Kerren A., North C., StuerZLINGER W.: Interaction for immersive analytics. In Immersive Analytics, Marriott K., Schreiber F., Dwyer T., Klein K., Riche N. H., Itoh T., Stuerzlinger W., Thomas B. H., (Eds.). Springer, Berlin/Heidelberg, 2018, ch. 4, pp. 95-138. doi: 10.1007/978-3-030-01388-2_4

[BD17] BESANÇON L., DRAGICEVIC P.: La différence significative entre valeurs $\mathrm{p}$ et intervalles de confiance (The significant difference between p-values and confidence intervals). In Conférence Francophone sur l'Interaction Homme-Machine (2017), AFIHM, Poitiers, France.

[BD19] BESANÇON L., DRAGICEVIC P.: The continued prevalence of dichotomous inferences at CHI. In CHI Extended Abstracts (2019), ACM, New York. doi: 10.1145/3290607.3310432

[Bes18] BesAnÇON L.: An Interaction Continuum for 3D Dataset Visualization. Theses, Université Paris-Saclay, 2018.

[BH09] Brewster S. A., Hughes M.: Pressure-based text entry for mobile devices. In Proc. MobileHCI (2009), ACM, New York, pp. 9:19:4. doi: $10.1145 / 1613858.1613870$

[BI12] Bezerianos A., IsEnBerg P.: Perception of visual variables on tiled wall-sized displays for information visualization applications. IEEE Transactions on Visualization and Computer Graphics 18, 12 (Dec 2012), 2516-2525. doi: 10.1109/TVCG.2012.251

[BIAI17a] BesAnÇOn L., IsSARTEl P., AMMi M., IsEnberg T.: Hybrid tactile/tangible interaction for 3D data exploration. IEEE Transactions on Visualization and Computer Graphics 23, 1 (Jan. 2017), 881-890. doi: 10.1109/TVCG.2016.2599217

[BIAI17b] BesAnÇON L., IsSARTEl P., AMmi M., Isenberg T.: Mouse, tactile, and tangible input for 3D manipulation. In Proc. CHI (2017), ACM, New York, pp. 4727-4740. doi: 10.1145/3025453.3025863

[BIRW19] BRUCKNER S., ISENBERG T., Ropinski T., Wiebel A.: A model of spatial directness in interactive visualization. IEEE Transactions on Visualization and Computer Graphics 25 (2019). To appear. doi: 10. 1109/TVCG.2018.2848906

[BLC*12] Boring S., Ledo D., Chen X. A., Marquardt N., TAng A., Greenberg S.: The fat thumb: Using the thumb's contact size for single-handed mobile interaction. In Proc. MobileHCI (2012), ACM, New York, pp. 39-48. doi: 10.1145/2371574.2371582

[BS71] Burgess T. D. G., SAles S. M.: Attitudinal effects of "mere exposure": A reevaluation. Journal of Experimental Social Psychology 7, 4 (July 1971), 461-472. doi: 10.1016/0022-1031(71)90078-3

[Bux86] BUXTON W.: Chunking and phrasing and the design of humancomputer dialogues. In Proc. IFIP World Computer Congress (1986), pp. 475-480. Republished in Readings in Human-Computer Interaction, Baecker R.M., Grudin J., Buxton W.A.S., Greenberg S., (Eds.). Morgan Kaufmann, 1995, pp. 494-499. doi: 10.1016/B978-0-08-051574-8.50051-0

[BWB06] Benko H., Wilson A. D., Baudisch P.: Precise selection techniques for multi-touch screens. In Proc. CHI (2006), ACM, New York, pp. 1263-1272. doi: 10.1145/1124772.1124963

[CDH11] Cohé A., DÈCle F., HAChet M.: tBox: A 3D transformation widget designed for touch-screens. In Proc. CHI (2011), ACM, New York, pp. 3005-3008. doi: 10.1145/1978942.1979387 
[CDVB17] CORSTEN C., DAEHLMANN B., VOELKER S., BORCHERS J.: BackXPress: Using back-of-device finger pressure to augment touchscreen input on smartphones. In Proc. CHI (2017), ACM, New York, pp. 4654-4666. doi: 10.1145/3025453.3025565

[CML*12] CofFey D., MalbraAten N., LE T., BoraZjani I., Sotiropoulos F., ERdman A. G., KeEFE D. F.: Interactive Slice WIM: Navigating and interrogating volume data sets using a multisurface, multitouch VR interface. IEEE Transactions on Visualization and Computer Graphics 18, 10 (Oct. 2012), 1614-1626. doi: 10.1109/TVCG.2011. 283

[CMS88] Chen M., Mountford S. J., Sellen A.: A study in interactive 3-D rotation using 2-D control devices. ACM SIGGRAPH Computer Graphics 22, 4 (Aug. 1988), 121-129. doi: 10.1145/378456.378497

[CNSD93] Cruz-Neira C., Sandin D. J., DeFanti T. A.: Surroundscreen projection-based virtual reality: The design and implementation of the CAVE. In Proc. SIGGRAPH (1993), ACM, New York, pp. 135-142. doi: $10.1145 / 166117.166134$

[CSH*14] Chen X. A., Schwarz J., Harrison C., MankofF J., HUDSON S. E.: Air+touch: Interweaving touch \& in-air gestures. In Proc. UIST (2014), ACM, New York, pp. 519-525. doi: 10.1145/2642918. 2647392

[Cum12] Cumming G.: Understanding The New Statistics: Effect Sizes, Confidence Intervals and Meta-Analysis. Routledge Taylor \& Francis Group, New York, 2012. doi: 10.4324/9780203807002

[Cum13] Cumming G.: Introduction to statistics 9: Dance of the $\mathrm{p}$ values. YouTube video: https://www. youtube.com/watch?v= 50L1RqHrZQ8, 2013.

[Cum14] Cumming G.: The new statistics: Why and how. Psychological Science 25, 1 (Jan. 2014), 7-29. doi: 10.1177/0956797613504966

[DCH14] Dragicevic P., Chevalier F., Huot S.: Running an HCI experiment in multiple parallel universes. In CHI Extended Abstracts (2014), ACM, New York, pp. 607-618. doi: 10.1145/2559206.2578881

[DJFW15] Deber J., Jota R., Forlines C., Wigdor D.: How much faster is fast enough?: User perception of latency \& latency improvements in direct and indirect touch. In Proc. CHI (2015), ACM, New York, pp. 1827-1836. doi: $10.1145 / 2702123.2702300$

[Dra16] DRAGICEVIC P.: Fair statistical communication in HCI. In Modern Statistical Methods for HCI, Robertson J., Kaptein M., (Eds.) Springer International Publishing, Cham, Switzerland, 2016, ch. 13, pp. 291-330. doi: 10.1007/978-3-319-26633-6_13

[DVM*12] Dell N., Vaidyanathan V., Medhi I., Cutrell E., THIES W.: "Yours is better!" Participant response bias in HCI. In Proc. CHI (2012), ACM, New York, pp. 1321-1330. doi: 10.1145/2207676. 2208589

[FGN10] FU C.-W., GoH W.-B., NG J. A.: Multi-touch techniques for exploring large-scale 3D astrophysical simulations. In Proc. CHI (2010), ACM, New York, pp. 2213-2222. doi: 10.1145/1753326.1753661

[FWSB07] FORLINES C., Wigdor D., SHEN C., BALAKRISHNAN R.: Direct-touch vs. mouse input for tabletop displays. In Proc. CHI (2007), ACM, New York, pp. 647-656. doi: 10.1145/1240624.1240726

[GBC13] Glesser D., BérARD F., CoOperstock J. R.: Overcoming limitations of the trackpad for 3D docking operations. In CHI Extended Abstracts (2013), ACM, New York, pp. 1239-1244. doi: 10.1145/2468356. 2468578

[GWP12] Goel M., Wobbrock J., Patel S.: Gripsense: Using built-in sensors to detect hand posture and pressure on commodity mobile phones. In Proc. UIST (2012), ACM, New York, pp. 545-554. doi: 10.1145/2380116. 2380184

[Har06] HART S. G.: NASA-task load index (NASA-TLX); 20 years later. Proceedings of the Human Factors and Ergonomics Society Annual Meeting 50, 9 (Oct. 2006), 904-908. doi: 10.1177/154193120605000909

[HBW13] HWANG S., BIANCHI A., WoHN K.-Y.: VibPress: Estimating pressure input using vibration absorption on mobile devices. In Proc. MobileHCI (2013), ACM, New York, pp. 31-34. doi: 10.1145/2493190.2493193
[HCC07] HANCOCK M., CARPENDAlE S., COCKBURN A.: Shallowdepth 3D interaction: Design and evaluation of one-, two- and three-touch techniques. In Proc. CHI (2007), ACM, New York, pp. 1147-1156. doi: 10.1145/1240624.1240798

[HCV*06] Hancock M. S., Carpendale S., Vernier F. D., WigDOR D., SHEN C.: Rotation and translation mechanisms for tabletop interaction. In Proc. TABLETOP (2006), IEEE Computer Society, Los Alamitos, pp. 79-88. doi: 10.1109/TABLETOP.2006.26

[HGB*06] Hinckley K., Guimbretiere F., Baudisch P., Sarin R., Agrawala M., Cutrell E.: The springboard: Multiple modes in one spring-loaded control. In Proc. CHI (2006), ACM, New York, pp. 181-190. doi: 10.1145/1124772.1124801

[HHP*16] Hinckley K., Heo S., Pahud M., Holz C., Benko H., Sellen A., Banks R., O’Hara K., Smyth G., Buxton W.: Pretouch sensing for mobile interaction. In Proc. CHI (2016), ACM, New York, pp. 2869-2881. doi: 10.1145/2858036.2858095

[Hib99] HibBaRd B.: Top ten visualization problems. ACM SIGGRAPH Computer Graphics 33, 2 (May 1999), 21-22. doi: 10.1145/326460.326485

[HL11a] HEO S., LEE G.: Force gestures: Augmented touch screen gestures using normal and tangential force. In CHI Extended Abstracts (2011), ACM, New York, pp. 1909-1914. doi: 10.1145/1979742.1979895

[HL11b] HEO S., LEE G.: Forcetap: Extending the input vocabulary of mobile touch screens by adding tap gestures. In Proc. MobileHCI (2011), ACM, New York, pp. 113-122. doi: 10.1145/2037373.2037393

[HL12] HEO S., LEE G.: Forcedrag: Using pressure as a touch input modifier. In Proc. OzCHI (2012), ACM, New York, pp. 204-207. doi: 10. $1145 / 2414536.2414572$

[HPGK94] Hinckley K., Pausch R., Goble J. C., Kassell N. F.: Passive real-world interface props for neurosurgical visualization. In Proc. CHI (1994), ACM, New York, pp. 452-458. doi: 10.1145/191666.191821

[HtCC09] Hancock M., TEN CATE T., CARPEndale S.: Sticky tools: Full 6DOF force-based interaction for multi-touch tables. In Proc. ITS (2009), ACM, New York, pp. 133-140. doi: 10.1145/1731903.1731930

[HW78] HEROT C. F., WEINZAPFEL G.: One-point touch input of vector information for computer displays. ACM SIGGRAPH Computer Graphics 12, 3 (Aug. 1978), 210-216. doi: 10.1145/965139.807392

[IBG*16] Issartel P., Besançon L., Guéniat F., Isenberg T., Ammi M.: Preference between allocentric and egocentric 3D manipulation in a locally coupled configuration. In Proc. SUI (2016), ACM, New York, pp. 79-88. doi: 10.1145/2983310.2985750

[IH12] IsENBERG T., HANCOCK M.: Gestures vs. postures: 'Gestural' touch interaction in 3D environments. In Proc. 3DCHI (2012), pp. 53-61.

[Ise16] IsENBERG T.: Interactive exploration of three-dimensional scientific visualizations on large display surfaces. In Collaboration Meets Interactive Spaces, Anslow C., Campos P., Jorge J., (Eds.). Springer, Berlin/Heidelberg, 2016, ch. 6, pp. 97-123. doi: 10.1007/978-3-319-45853-3_6

[JDF12] Jansen Y., DragiCeViC P., FeKete J.-D.: Tangible remote controllers for wall-size displays. In Proc. CHI (2012), ACM, New York, pp. 2865-2874. doi: 10.1145/2207676.2208691

[Joh04] JoHnson C. R.: Top scientific visualization research problems. IEEE Computer Graphics and Applications 24, 4 (July/Aug. 2004), 13-17. doi: 10.1109/MCG.2004.20

[KA13] KRZYWinski M., Altman N.: Points of significance: Error bars. Nature Methods 10, 10 (Oct. 2013), 921-922. doi: 10.1038/nmeth.2659

[KCST05] Kruger R., Carpendale S., Scott S. D., TAng A.: Fluid integration of rotation and translation. In Proc. CHI (2005), ACM, New York, pp. 601-610. doi: 10.1145/1054972.1055055

[Kee10] KEEFE D. F.: Integrating visualization and interaction research to improve scientific workflows. IEEE Computer Graphics and Applications 30, 2 (Mar. 2010), 8-13. doi: 10.1109/MCG.2010.30

[KH11] KNOEDEL S., Hachet M.: Multi-touch RST in 2D and 3D spaces: Studying the impact of directness on user performance. In Proc. 
3DUI (2011), IEEE Computer Society, Los Alamitos, pp. 75-78. doi: 10. 1109/3DUl.2011.5759220

[KI13] KeEFE D. F., IsenberG T.: Reimagining the scientific visualization interaction paradigm. IEEE Computer 46, 5 (May 2013), 51-57. doi: 10.1109/MC.2013.178

[KKC $\left.{ }^{*} 15\right]$ KIM K., KIM J., ChOI J., KIM J., LeE S.: Depth camerabased 3D hand gesture controls with immersive tactile feedback for natural mid-air gesture interactions. Sensors 15, 1 (2015), 1022-1046. doi: 10. 3390/s150101022

[LAFT12] LiU J., Au O. K.-C., FU H., TAI C.-L.: Two-finger gestures for 6DOF manipulation of 3D objects. Computer Graphics Forum 31 7pt1 (Sept. 2012), 2047-2055. doi: 10.1111/j.1467-8659.2012.03197.x

[Lai10] LAI J.: Dichotomous thinking: A problem beyond NHST. In Proc. ICOTS 8 (2010), International Association for Statistical Education, Voorburg, the Netherlands, pp. C101:1-C101:4.

[LHD17] LANGNER R., HORAK T., DACHSElT R.: VisTiles: Coordinating and combining co-located mobile devices for visual data exploration. IEEE Transactions on Visualization and Computer Graphics 24, 1 (Jan. 2017), 626-636. doi: 10.1109/TVCG.2017.2744019

[LKM*17] LaViola JR. J. J., KruijfF E., MCMahan R. P., Bowman D. A., PoupyreV I.: 3D User Interfaces: Theory and Practice, $2^{\text {nd }}$ ed Addison-Wesley, Boston, 2017.

[LODI16] López D., Oehlberg L., Doger C., Isenberg T.: Towards an understanding of mobile touch navigation in a stereoscopic viewing environment for 3D data exploration. IEEE Transactions on Visualization and Computer Graphics 22, 5 (May 2016), 1616-1629. doi: 10.1109/TVCG 2015.2440233

[LRF* 11] Lundström C., Rydell T., Forsell C., Persson A. YNNERMAN A.: Multi-touch table system for medical visualization: Application to orthopedic surgery planning. IEEE Transactions on Visualization and Computer Graphics 17, 12 (Dec. 2011), 1775-1784. doi: 10. 1109/TVCG.2011.224

[MCG10] Martinet A., Casiez G., Grisoni L.: The effect of DOF separation in 3D manipulation tasks with multi-touch displays. In Proc. VRST (2010), ACM, New York, pp. 111-118. doi: 10.1145/1889863.1889888

[Mun14] Munzner T.: Visualization Analysis and Design. CRC Press, Boca Raton, FL, USA, 2014. doi: 10.1201/b17511

[MZ99] MacKenZIE I. S., Zhang S. X.: The design and evaluation of a high-performance soft keyboard. In Proc. CHI (1999), ACM, New York, pp. 25-31. doi: 10.1145/302979.302983

[NBBW09] Nacenta M. A., Baudisch P., Benko H., Wilson A. Separability of spatial manipulations in multi-touch interfaces. In Proc Graphics Interface (2009), Canadian Information Processing Society, Toronto, pp. 175-182. doi: 10.20380/GI2009.23

[NFRCC05] Noirhomme-Fraiture M., Randolet F., Chittaro L., Custinne G.: Data visualizations on small and very small screens. In Proc. Symposium on Applied Stochastic Models and Data Analysis (2005), ENST, France, pp. 276-285.

[OO05] OAKLEY I., O'MODHRAIN S.: Tilt to scroll: Evaluating a motion based vibrotactile mobile interface. In Proc. World Haptics (March 2005), IEEE Computer Society, Los Alamitos, pp. 40-49. doi: 10.1109/WHC.2005. 138

[PM03] Poupyrev I., MARUYAMA S.: Tactile interfaces for small touch screens. In Proc. UIST (2003), ACM, New York, pp. 217-220. doi: 10 $1145 / 964696.964721$

[PN16] PELURSON S., NigAY L.: Bimanual input for multiscale navigation with pressure and touch gestures. In Proc. ICMI (2016), ACM, New York, pp. 145-152. doi: 10.1145/2993148.2993152

[POR*18] Pahud M., Ofek E., Riche N. H., Hurter C., Grubert J.: Mobiles as Portals for Interacting with Virtual Data Visualizations. arXiv pre-print 1804.03211, Apr 2018.

[QM05] QI W., MARTENS J.-B.: Tangible user interfaces for 3D clipping plane interaction with volumetric data: A case study. In Proc. ICMI (2005), ACM, New York, pp. 252-258. doi: 10.1145/1088463.1088507
[QTHM06] QI W., TAYLOR II R. M., HEALEY C. G., MARTENS J.-B.: A comparison of immersive HMD, fish tank VR and fish tank with haptics displays for volume visualization. In Proc. APGV (2006), ACM, New York, pp. 51-58. doi: 10.1145/1140491.1140502

[RBB04] Ramos G., Boulos M., Balakrishnan R.: Pressure widgets. In Proc. CHI (2004), ACM, New York, pp. 487-494. doi: 10.1145/ 985692.985754

[RDH09] REISMAN J. L., DAVIDSON P. L., HAN J. Y.: A screen-space formulation for 2D and 3D direct manipulation. In Proc. UIST (2009), ACM, New York, pp. 69-78. doi: 10.1145/1622176.1622190

[RGB*10] Raynal M., Gauffre G., BaCh C., Schmitt B., Dubois E.: Tactile camera vs. tangible camera: Taking advantage of small physical artefacts to navigate into large data collection. In Proc. NordiCHI (2010), ACM, New York, pp. 373-382. doi: 10.1145/1868914.1868958

[Rhe02] Rheingans P.: Are we there yet? Exploring with dynamic visualization. IEEE Computer Graphics and Applications 22, 1 (Jan. 2002), 6-10. doi: 10.1109/38.974511

[RLG09] Roudaut A., Lecolinet E., Guiard Y.: Microrolls: Expanding touch-screen input vocabulary by distinguishing rolls vs. slides of the thumb. In Proc. CHI (2009), ACM, New York, pp. 927-936. doi: 10.1145/1518701.1518843

[SBD12] SPINDLER M., BÜSCHEL W., DACHSElT R.: Use your head: Tangible windows for 3D information spaces in a tabletop environment In Proc. ITS (2012), ACM, New York, pp. 245-254. doi: 10.1145/2396636. 2396674

[SFH*02] Schmalstieg D., Fuhrmann A., Hesina G., Szalavár Z., Encarnaç̈o L. M., Gervautz M., Purgathofer W.: The Studierstube augmented reality project. Presence: Teleoper. Virtual Environ. 11, 1 (Feb. 2002), 33-54. doi: 10.1162/105474602317343640

[Sha98] SHAw C. D.: Pain and fatigue in desktop VR: Initial results. In Proc. Graphics Interface (1998), Canadian Information Processing Society, Toronto, pp. 185-192. doi: 10.20380/Gl1998.23

[SKB92] Sellen A. J., Kurtenbach G. P., Buxton W. A. S.: The prevention of mode errors through sensory feedback. Human Computer Interaction 7, 2 (June 1992), 141-164. doi: 10.1207/s15327051hci0702 1

[SL10] SAURo J., LEWIS J. R.: Average task times in usability tests: What to report? In Proc. CHI (2010), ACM, New York, pp. 2347-2350. doi: $10.1145 / 1753326.1753679$

[SRE17] SCHWANK J., RUPPRECHT F.-A., EBERT A.: Waggle Orientation-based tablet interaction. In CHI Extended Abstracts (2017), ACM, New York, pp. 2042-2048. doi: 10.1145/3027063.3053151

[Sut66] Sutherland I. E.: Ten unsolved problems in computer graphics. Datamation 12, 5 (1966), 22

[SvZP*16] Sollich H., von Zadow U., Pietzsch T, TOMANCAK P. DACHSELT R.: Exploring time-dependent scientific data using spatially aware mobiles and large displays. In Proc. ISS (2016), ACM, New York, pp. 349-354. doi: 10.1145/2992154.2996779

[TM04] TORY M., MÖLlER T.: Human factors in visualization research. IEEE Transactions on Visualization and Computer Graphics 10, 1 (Jan./Feb. 2004), 72-84. doi: 10.1109/TVCG.2004.1260759

[Tuk77] Tukey J. W.: Exploratory Data Analysis. Pearson, Reading, Mass., 1977.

[VAl15] VAlentine J. C., Aloe A. M., LAU T. S.: Life after NHST How to describe your data without " $p$-ing" everywhere. Basic and Applied Social Psychology 37, 5 (Aug. 2015), 260-273. doi: 10.1080/01973533.2015. 1060240

[Van09] VandenBos G. R. (Ed.): Publication Manual of the American Psychological Association, $6^{\text {th }}$ ed. APA, Washington, DC, 2009.

[VCB09] VEIT M., CAPOBIANCO A., BECHMANN D.: Influence of degrees of freedom's manipulation on performances during orientation tasks in virtual reality environments. In Proc. VRST (2009), ACM, New York, pp. 51-58. doi: 10.1145/1643928.1643942 
[VSC15] Vuibert V., Stuerzlinger W., Cooperstock J. R.: Evaluation of docking task performance using mid-air interaction techniques. In Proc. SUI (2015), ACM, New York, pp. 44-52. doi: 10.1145/2788940. 2788950

[WBG*19] Wang X., Besançon L., Guéniat F., Sereno M., Ammi M., ISENBERG T.: A vision of bringing immersive visualization to scientific workflows. In Proc. Workshop on Immersive Analytics (CHI-IA) (2019). To appear.

[WPSN15] Withana A., PeIRIS R., SAMARASEKARA N., NANAYAKKARA S.: zSense: Enabling shallow depth gesture recognition for greater input expressivity on smart wearables. In Proc. CHI (2015), ACM, New York, pp. 3661-3670. doi: 10.1145/2702123.2702371

[WSB10] Wilson G., Stewart C., Brewster S. A.: Pressure-based menu selection for mobile devices. In Proc. MobileHCI (2010), ACM, New York, pp. 181-190. doi: 10.1145/1851600.1851631

[WWX*18] WANG L., Wong L., Xu Y., Zhou X., QIU S., Meng $X$. , YANG C.: The design and empirical evaluations of 3D positioning techniques for pressure-based touch control on mobile devices. Personal Ubiquitous Computing 22, 3 (June 2018), 525-533. doi: 10.1007/s00779-018 $-1147-0$

[YKS07] Yi J. S., KANG Y. A., STASKO J.: Toward a deeper understanding of the role of interaction in information visualization. IEEE Transactions on Visualization and Computer Graphics 13, 6 (Nov 2007), 1224-1231. doi: 10.1109/TVCG.2007.70515

[YSI*10] Yu L., SVETACHOV P., ISENBERG P., EVERTS M. H., ISENBERG T.: FI3D: Direct-touch interaction for the exploration of 3D scientific visualization spaces. IEEE Transactions on Visualization and Computer Graphics 16, 6 (Nov./Dec. 2010), 1613-1622. doi: 10.1109/TVCG. 2010.157

[ZF99] ZELEZNIK R., Forsberg A.: Unicam-2D gestural camera controls for 3D environments. In Proc. I3D (1999), ACM, New York, pp. 169-173. doi: $10.1145 / 300523.300546$

[Zha98] ZHAI S.: User performance in relation to 3D input device design. ACM SIGGRAPH Computer Graphics 32, 4 (Nov. 1998), 50-54. doi: 10. $1145 / 307710.307728$

[ZS97a] ZHAI S., SENDERS J. W.: Investigating coordination in multidegree of freedom control I: Time-on-target analysis of 6 DOF tracking. Proceedings of the Human Factors and Ergonomics Society Annual Meeting 41, 2 (Oct. 1997), 1249-1253. doi: 10.1177/1071181397041002117

[ZS97b] ZhAI S., SENDERS J. W.: Investigating coordination in multidegree of freedom control II: Correlation analysis in 6 DOF tracking. Proceedings of the Human Factors and Ergonomics Society Annual Meeting 41, 2 (Oct. 1997), 1254-1258. doi: 10.1177/1071181397041002118 